\title{
Molecular Simulations in Materials Science
}

\section{SUN Huai}

School of Chemistry and Chemical Engineering, Shanghai Jiao Tong University, Shanghai 200240, P. R. China.

Molecular simulation finds application in a wide range of research fields based on life and materials sciences. It helps comprehend and predict the chemical and physical properties of substances; thus, it is useful in directing R\&D and industrial production. In this special issue, we focus on molecular simulations in material sciences.

Molecular simulation employs computational models from microscopic to mesoscopic levels, which is reflected in this special issue. For example, Liu et al. ${ }^{1}$ reported modulation of catalytic activity for $\mathrm{CO}_{2}$ hydrogenation using quantum density functional theory (DFT). Yin et al. ${ }^{2}$ parameterized a semiempirical density functional tight-binding (DFTB) model to study deposition of carbon on copper surface. At the atomic level, Ren et al. ${ }^{3}$ simulated the thermal decomposition of highenergy-density materials using reactive force field, and Wang et al. ${ }^{4}$ reported the use of a flexible and polarizable force field to calculate vibrational frequencies of molecules on liquid surface. Combining DFT calculations and atomistic force field, Liu et al. ${ }^{5}$ studied the effect of photoisomerization on binding energy and conformation of azobenzene-containing host-guest complex. At the mesoscopic level, Lu et al. ${ }^{6}$ studied the deformation of polymer-grafted Janus nanosheets using the dissipative particle dynamics (DPD) model.

Developing new methods to enhance simulation efficiency has always been at the center of molecular simulation. One such example reported herein by Xue et al. ${ }^{7}$, is a model that minimizes the number of explicit solvent molecules in the quantum chemistry calculation of vibrational spectra. In recent years, research on free energy calculation and enhanced sampling technique has advanced considerably. In this special issue, Yoshii et al. ${ }^{8}$ tackled the time-scale problem of simulating the self-assembly of ionic surfactants in aqueous solution by calculating the free energy changes. Meta-dynamics, one of the advanced free energy calculation methods, is used by Yin et al. ${ }^{2}$ to calculate the free energy change associated with carbon dimerization on copper (111) surface. Xin and Sun ${ }^{9}$ reported their research on replica exchange methods in the simulation of complex reactions characterized by coexistence of hundred elemental reactions.

Another essential factor in molecular simulations is the potential energy function or what is commonly called "force field". In this special issue, several groups have reported their studies on force field development. In order to predict IR, Raman and sum frequency generation spectra of ethylene carbonate at liquid-vapor interface with precision comparable with experimental measurements, a sophisticated and polarizable force field has been developed by Wang et al. ${ }^{4}$ Their predictions provide detailed description of the surface configuration, which is inaccessible experimentally. At the semi-empirical level, Yin et al. ${ }^{2}$ parameterized a tight-binding model to describe the carbon-copper and carbon-carbon interactions based on DFT calculations. Liu et al. ${ }^{5}$ parameterized an all-atom force field for azobenzene-containing complexes, and $\mathrm{Lu}$ et al. ${ }^{6}$ compared different sets of interaction parameters in their dissipative particle dynamics simulations.

The articles presented in this special issue demonstrate the roles played by molecular simulation in the cutting edge of research and development. The topics covered include $\mathrm{CO}_{2}$ reduction ${ }^{1}$, molecular machinery ${ }^{5}$, graphene chemistry ${ }^{10}$, carbon deposition on metal surface ${ }^{2}$, complex reactions ${ }^{3,9}$, surface chemistry ${ }^{4}$, polymer morphology ${ }^{6}$, and self-assembly ${ }^{8}$. Although the collection of articles is far from complete in reflecting the research activities, we hope that it is helpful especially to those researchers who are new to the field to get a snapshot of what can be achieved by using molecular simulations in materials research.

\section{References}

(1) Liu, T.; Li, J.; Liu, W. J.; Zhu, Y. D.; Lu, X, H. Acta Phys. -Chim. Sin. 2018, 34, 1097. doi: 10.3866/PKU.WHXB201712131

(2) Yin, D.; Qiu, Z. Y.; Li, P.; Li, Z. Y. Acta Phys. -Chim. Sin. 2018, 34, 1116. doi: 10.3866/PKU.WHXB201801151

(3) Ren, C. X.; Li, X. X.; Guo, L. Acta Phys. -Chim. Sin. 2018, 34, 1151. doi: 10.3866/PKU.WHXB201802261

(4) Wang, L.; Xin, L.; Ishiyama, T.; Peng, Q. L.; Ye, S.; Morita, A. Acta Phys. -Chim. Sin. 2018, 34, 1124. doi: 10.3866/PKU.WHXB201801291

(5) Liu, P. Y.; Liu, C. Y.; Liu, Q.; Ma, J. Acta Phys. -Chim. Sin. 2018, 34, 1171. doi: 10.3866/PKU.WHXB201803024

(6) Lu, T.; Zhou, Y. X.; Guo, H. X. Acta Phys. -Chim. Sin. 2018, 34 , 


\section{4. doi: 10.3866/PKU.WHXB201802122}

(7) Xue, B.; Chen, T. N.; Siepmann, J. I. Acta Phys. -Chim. Sin. 2018, 34, 1106. doi: 10.3866/PKU.WHXB201701083

(8) Yoshii, N.; Komori, M.; Kawada, S.; Takabayashi, H.; Fujimoto, K.; Okazaki, S. Acta Phys. -Chim. Sin. 2018, 34, 1163. doi: 10.3866/PKU.WHXB201802271

(9) Xin, L.; Sun, H. Acta Phys. -Chim. Sin. 2018, 34, 1179. doi: 10.3866/PKU.WHXB201803161

(10) Sun, C. Z.; Bai, B. F. Acta Phys. -Chim. Sin. 2018, 34, 1136. doi: 10.3866/PKU.WHXB201801301 